\title{
Analysis of the Communication Demand of Smart Grid
}

\author{
Yongbo Yang, Cheng Zhang, Liqiang Lu, Ruihua Ren, Tao Fu, Xin Chen, Jia \\ Sun and Bo Kong
}

\author{
State Grid Henan Electric Power Company Zhengzhou Electric Power Supply Company Zhengzhou \\ Henan, China
}

Keywords: Smart grid; Communication technology; AMI; SCADA

\begin{abstract}
Smart grid has become the hot topic to domestic and foreign about the development trend of power grid in recent years, and communication technology is foundation of the development of smart grid; the paper forecasts the smart grid communications automation and development trend. Further from intelligent dispatching center, smart grid, smart distribution grid and users, in view of the existing and emerging intelligent and automatic application were communication needs analysis, and provide a reliable basis for the smart grid communications planning.
\end{abstract}

\section{Introduction}

Research shows that communication is the foundation of smart grid project, and it is indispensable for any smart grid [1]. In these following smart grid functions, which is particularly evident. Control center of communication need to bring the latest data back [2]. Intelligent feeder switches need to exchange information with each other, and the control center upload data, so that the control center to coordinate, effective work. The regulator and reactive power compensation in the control function of the advanced reactive power compensation need to exchange data with each other, and upload control center to coordinate and control the voltage. Advanced Metering Infrastructure, AMI, demand response, communication transmission demand response signal, return measurement results and verify the load changes. Supervisory control and data acquisition, SCADA, requires the communication network between control equipment and the control center, as well as the devices [3].

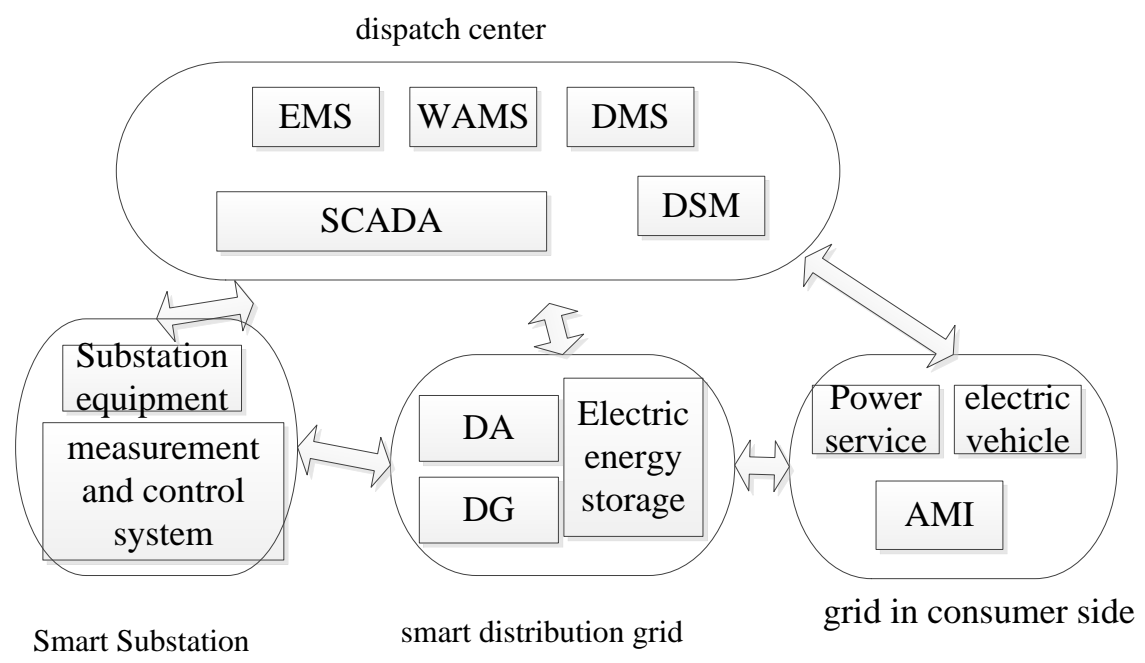

Figure 1. Smart grid and communication

As clear from the unique role of communication in smart grid, technology is the foundation of the realization of other technology. Full implementation of the smart grid, promote the communication demand, may need 10000 to 100000 times the amount of data of the current power grid. 


\section{Requirements for Communication in Smart Grid Construction}

The construction of smart grid requires rigorous planning, and its design and planning face many challenges.

The first is the support function of a single communication system to multiple smart grid. For example, a single network can support two kinds of functions, such as prepayment metering and demand response.

Second is the communication technology itself is a development of technology. Communication infrastructure update, communication technology is also changing with each passing day.

Third is the smart grid functions are also rapidly developing. Social innovation accelerates, the power system in order to reduce system loss, better integration of renewable energy, improve reliability, each day has a new idea generation. The vast majority of these new features require bandwidth support, will be a serious test of the communication network.

How to build a communication system to support all of the current and future smart grid needs is the most important issue in the construction of communication networks. The answer will be different because of the terrain, the grid configuration, the environment, the density of the electric meter and so on. But need to analyze the current and future communication needs for smart grid communications construction guidelines.

Is proposed in this paper on smart grid communications automation and development trend were analyzed (the intelligent power system without further analysis), and analysis of the existing and emerging intelligent application, such as SCADA, DA, AMI communication needs.

\section{Development Trend of Intelligent Network and Communication}

In the past, the implementation of new applications, such as SCADA or AMI, must invest in new communications infrastructure. Because the technology is not mature, the old communication network is mainly used for single application. Today, however, the State Grid Corporation is building a more powerful communication network, can be gradually extended to add new applications and existing applications running at the same time. Because most of the new communications infrastructure to support multiple power grid applications, but also because the construction of the infrastructure is currently more expensive, making the risk of false selection is also large. In order to reduce this risk, the future strategic planning should be determined in advance for the automation and communications of the entire power industry. The smart grid is divided into control center, intelligent transmission and distribution grid, smart substation, intelligent power grid in order to analyze.

Intelligent Dispatch Center. Monitoring center communication network. There are many nodes in the power network, which connect to the monitoring center through the data network. Monitoring center installed customer information system (CIS), AMI, OMS and the host of the telephone system, etc., these data network bandwidth is often insufficient. This situation may be due to further implementation of cloud technology (or "private cloud") resulting in a greater shortage of. Therefore, the prediction of the bandwidth requirements must be part of the long-term planning.

The impact of cloud computing. More and more applications are now available through the provider of cloud based software solutions. "Cloud based" generally refers to the use of "public cloud" in a central data center remote operation and through the public Internet access software. Now, as cloud based software such as instrumentation data management system (MDMS) and other software applications, the Internet is becoming more and more critical than in the past. Thanks to cloud based software, the Internet is becoming more and more important in comparison to the past. So we need to evaluate the impact on the future of intelligent applications based on cloud computing.

Smart Substation. Intelligent substation is using the advanced, reliable, integrated and environmentally friendly intelligent equipment, to the total information digitization, communication platform networking, information sharing standardization for the basic requirements, automatic completion of the basic functions of information collecting, measuring, control, protection, measurement and testing, at the same time, with the support of real-time automatic control, intelligent 
regulation, online analysis and decision and collaborative interaction and other advanced features of the substation[4].

Change of SCADA in substation. If it is now part of the city does not have the function of SCADA, or the existing SCADA function improvement, such as from the development of proprietary protocols for distributed network protocol / Internet Protocol (DNP/ IP) may in substation called for the establishment of new communication facilities. Substation automation system, refers to the gradual upgrading of electronic equipment for the substation, which mainly includes a remote access to intelligent devices, provides a two-way data flow. The system needs to provide high reliability of the communication, and is highly redundant. The system also provides the function of the control circuit breaker, and as the historical data and start the reactive power automatic compensation device. If the reliability of the system is not enough, it can cause the failure of the circuit breaker, causing the external power failure, which greatly reduces the reliability of the system. The data transmission frequency must be kept in a high range, the data delay must be kept in the millisecond level, in order to ensure the real-time performance and reliability of the monitoring system. Communication architecture in the substation as shown in Fig. 2.

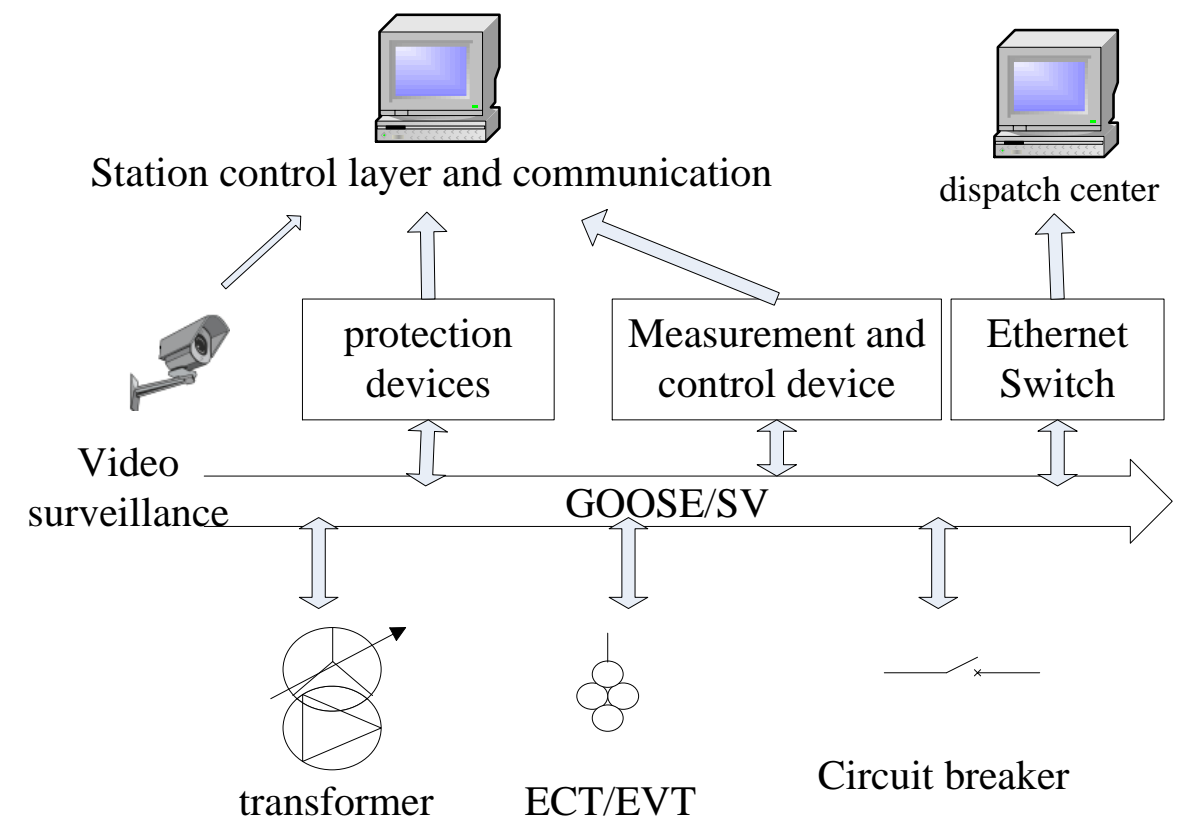

Figure 2. Communication architecture in Substation

AMI return based on PLC in a substation [5].In the existing automation measurement, if the AMI is not in place, or there is a traditional automatic meter reading (AMR) system in the application, it may require a new return communication network to meet the communication needs of AMI system. Automatic meter data from the substation PLC automatic device through the communication network to the Power Grid Corp control center to send the initiative, frequency from every 5 minutes to once a day, and even once a week. According to the experience, the general rule is that a single measurement data represents about 50 to 100 bytes (different from the vendor), including the original data and the IP TCP/ address. Therefore, the measurement data is a key variable in determining the size of the data file. In addition, the AMI system will be used for a variety of temporary requests, such as personal meter reading, power failure detection and recovery, load control events, prepayment meter reading and voltage reading.

Intelligent auxiliary control system. Intelligent auxiliary control system is an important aspect of the development of intelligent substation, which is the intelligent control system of the transformer substation. Including the substation fire control system, video surveillance system, access control system, etc.. For substation intelligent construction, substation operation and maintenance of unattended mode, operation and maintenance work efficiency, provides a reliable guarantee. But 
smart substation auxiliary control system due to the participation of the real-time video surveillance, a surge in the amount of data, video data of a current transformer substation often locally stored background monitoring machine. Video communication is not high requirements for the reliability of the system, does not affect the quality of monitoring fer is not the case. However, when the need for video transmission, when the remote real-time monitoring, data transmission must be maintained at a higher rate.

Smart Distribution Grid. China's current smart grid development mainly concentrated in UHV network, and North American countries mainly emphasizes the rapid development of smart distribution network, in our country under a five-year plan, will also be the development of distribution network as a key point. At the same time relative to the high voltage network, distribution network equipment and different, kinds of miscellaneous. Therefore, the development of smart distribution network will of communication network proposed higher requirements.

The further development of demand side management (DSM) program. One way $150 \mathrm{MHz}$ communication technology and PLC technology have been used for load control. However, the use of a variety of technologies to upload demand side data. Therefore, remote data acquisition may affect the demand for remote communication.

Smart meter on the user side. Smart meter based on fixed wireless AMI return, one of the main advantages of a fixed wireless AMI is to through the AMI network more rapid transmission of measurement data, from the instrument faster response interrupt and transmitted to the host control without polling mechanism, greatly improves the work efficiency. But some customer groups of measurement data backup more frequently, such as timing users, prepaid users and distributed generation (Generation Distributed) measurement.

Application of feeder distribution automation control and monitoring. This general term refers to a variety of distribution feeder program. Almost all of the DA schemes include a bidirectional communication between the control device and the SCADA host computer. Most Power Grid Corp to deploy DA have a more long-term plan, often in the same area with dozens of DA point of communication, so to ensure that the choice of communication technology is particularly important in the scalability. The latest research on intelligent distribution network focuses on some of the most common DA schemes: intelligent feeder switch, CVR and reactive power automatic compensation device.

Distributed relay protection DA[6].North America, some of the Power Grid Corp are deploying some type of DG program. The DTT scheme usually involves the communication between three points: the DG source, the relay located at the interface point, and the second relay located upstream close to the service station. The communication between relays is point to point.

In addition, the China State Grid Corp needs to establish a smart grid cannot be ignored an important requirement: security. With the development of network attack technology, communication facilities must pay attention to the communication security, especially the security data transmission. Even if the communication device does not have a built-in security mechanism, a variety of encryption mechanisms, such as SSL, certificates, and virtual private networks (VPN), can also be used to secure data on the network.

\section{Communication Infrastructure Requirements}

In the design of communication for each intelligent application, we should consider the operational requirements of the application according to the actual needs. It is clear that any application of "operational requirements", which will be closely related to the communication infrastructure. For example, the AMI data acquisition frequency in the acquisition center is improved, such as from once a week to once a day, 24 times a day, or once every 15 minutes? How many seconds of delay can be accepted, from the data sent to when receiving? All of these decisions will affect the requirements for the development of the communication system. 
The communication requirements of intelligent applications include: data throughput and delay, data transmission frequency, interface, reliability, standby power, network topology, jitter, etc.. In the intelligent application of communication planning, the above characteristics are analyzed, and the development of relevant requirements in order to guide the planning of communication infrastructure.

Typical requirements for all communications are determined by the application, and these requirements are raised as an overview. Terrain, operational preferences, budget, main system software features, device density, and other various factors, will always affect the unique development needs of each application. Typical requirements for some applications are shown in the Table 1.

Table 1 Intelligent application of communication demand table

\begin{tabular}{|l|l|l|l|l|l|}
\hline NO. & $\begin{array}{l}\text { Intelligent } \\
\text { application }\end{array}$ & $\begin{array}{l}\text { Data } \\
\text { quantity }\end{array}$ & Delay & Reliability & $\begin{array}{l}\text { Transmission } \\
\text { frequency }\end{array}$ \\
\hline 1 & AMI & $1 \mathrm{Mb} / \mathrm{p}$ & $60 \mathrm{~s}$ & $99.9 \%$ & $1 \mathrm{~h}-6 \mathrm{~h}$ \\
\hline 2 & SCADA & $4 \mathrm{~kb}$ & $150 \mathrm{~ms}$ & $99.999 \%$ & $2 \mathrm{~s}-5 \mathrm{~s}$ \\
\hline 3 & DA & $4 \mathrm{~kb} / \mathrm{DNP} 3$ & $1 \mathrm{~s}$ & $99.99 \%$ & $5 \mathrm{~s}-10 \mathrm{~s}$ \\
\hline 4 & MWM & $10 \mathrm{~kb}$ & $10 \mathrm{~s}$ & $99.9 \%$ & $5 \mathrm{~min}-10 \mathrm{~min}$ \\
\hline 5 & $\begin{array}{l}\text { Video } \\
\text { surveillance }\end{array}$ & $80 \mathrm{kbps}$ & real time & $99 \%$ & real time \\
\hline
\end{tabular}

\section{Summary}

Smart grid development is a slow process, in this process, there will be a variety of advanced applications, in order to meet the needs of intelligent power grid. And intelligent demand, often through the interaction of information to achieve, which requires further development of the communication network. For the reliable operation of the power grid to ensure, to ensure good economic benefit, the power of sustainable development, this paper on the current smart grid has been applied, and emerging technologies are analyzed in order to provide reference for the design of the communication system.

The development of smart grid will promote the innovation of information and communication network technology. In the near future, the telecommunications industry, the Internet industry, the power industry mutual ask will produce a huge "chemical reaction", for people's lives to provide greater convenience and support. In the process of building a smart grid with Chinese characteristics, in order to provide opportunities for power supply enterprises to further development, but also on the power supply enterprises put forward a huge challenge.

\section{References}

[1] Tsoukalas L.H. Gao R. From Smart Grids to an Energy Internet: Assumptions, Architectures and Requirements. Electric Utility Deregulation and Restructuring and Power Technologies, 2008. DRPT 2008. Third International Conference on 6-9 April 2008 :94-98.

[2] King R L. Information services for smart grids[C] Proceedings of IEEE PES General Meeting. Pittsburgh, PA, US A: IEEE, 2008:1-5.

[3] MOMOH J A. Smart Grid Design for Efficient and Flexible Power Networks Operation and Control Power Systems Conference and Exposition[C] 2009, IEEE/PES, 2009: 1-8.

[4] EPRI. Integration of advanced automation and enterprise information infrastructures: Harmonization of IEC 61850 and IEC 61970/61968 models[R]. USA EPRI Technical Report, Palo Alto, 2006. 
[5] BENNETT C, HIGHFILL D. Networking AMI Smart Meters[C] Energy 2030 Conference,2008. ENERGY 2008. IEEE17-18

[6] EPRI. Technical and System Requirements of Advanced DistributionAutomation [R]. 1010915. Palo Alto, CA, USA: EPRI, 2004. 